\title{
Higher Admission D-Dimer Values Are Associated With an Increased Risk of Nonroutine Discharge in Neurosurgery Patients
}

Michael Karsy ${ }^{1}$, Robert Kim ${ }^{1}$, Mohammed Azab ${ }^{1}$, Jonathan Harper ${ }^{1}$, Jian Guan ${ }^{1}$, Ilyas Eli ${ }^{1}$, William Couldwell ${ }^{1}$

1. Neurosurgery, University of Utah, Salt Lake City, USA

Corresponding author: William Couldwell,william.couldwell@hsc.utah.edu

\section{Abstract \\ Background}

D-dimers are serum acute-phase proteins with a role in mediating inflammation that may be used as biomarkers for the prediction of deep vein thrombosis. Recent studies have shown that D-dimers can be used to predict prognosis and stratify risk in neurosurgical patients; however, a comparative analysis across diagnostic subtypes has yet to be performed.

\section{Methods}

A bioinformatics analysis evaluated neurosurgical patients with admission D-dimer levels between 2008 and 2017. Nonroutine disposition (e.g., skilled nursing facility, rehabilitation, other hospital, mortality) was primarily evaluated.

\section{Results}

A total of 1,854 patients (mean age $55.1 \pm 18.2$ years, $55.4 \%$ male; mean admission D-dimer $4.83 \pm 7.78 \mu \mathrm{g} / \mathrm{ml}$ ) were identified. Patient diagnoses included vascular (27.1\%), trauma (16.4\%), multiple diagnoses (15.7\%), spine (15.6\%), tumor (13.0\%), and other (12.2\%) causes. Univariate analysis showed that older age $(\mathrm{p}=0.0001)$, higher American Society of Anesthesiologists (ASA) score $(\mathrm{p}=0.0001)$, lower Glasgow Coma Scale (GCS) score $(\mathrm{p}=0.0001)$, diagnosis type $(\mathrm{p}=0.0001)$, longer length of stay (LOS) $(\mathrm{p}=0.0001)$, higher infection rate $(p=0.0001)$, surgery in the past year $(p=0.02)$, and higher D-dimer levels $(3.4 \pm 4.9 \mathrm{vs.} 5.4 \pm 8.7 \mu \mathrm{g} / \mathrm{ml}$, $\mathrm{p}=0.0001$ ) were associated with nonroutine disposition. Multivariate logistic regression showed that elevated D-dimers were independently associated with a greater relative risk of nonroutine disposition (relative risk [RR] 1.026, 95\% CI 1.02-1.033, $\mathrm{p}=0.0001$ ).

\section{Conclusions}

Received 03/11/2020 Review began 03/18/2020 Review ended 07/13/2020 Published 07/27/2020

๑) Copyright 2020 Karsy et al. This is an open access article distributed under the terms of the Creative Commons Attribution License CC-BY 4.0., which permits unrestricted use, distribution, and reproduction in any medium, provided the original author and source are credited.
Elevated admission D-dimer values were independently associated with a $3 \%$ increased risk of nonroutine disposition per D-dimer unit after accounting for other factors. These results suggest that D-dimer values may help in stratifying patient risk models despite clinical heterogeneity. Further refinement of neurosurgical patient risk models using clinical variables and biomarkers may aid in resource allocation and early warning.

Categories: Neurosurgery

Keywords: d-dimer, biomarker, outcomes, neurosurgery

\section{Introduction}

The use of biomarkers to predict neurosurgical patient prognosis remains an active area of interest because it may enable better targeted therapies and allocation of resources to patients, as well as guidance to patients and providers. D-dimers are fibrin degradation products released into the bloodstream after blood clot fibrinolysis that have classically been used for the evaluation of venous thromboembolism [1,2]. However, D-dimers are also serum acute-phase proteins (APP) that show upregulated expression after stress, infection, or worsening disease states. The recent literature has suggested that D-dimers can be used to evaluate and predict clinical prognosis in neurosurgical patients, including after subarachnoid hemorrhage [3-6], intracranial hemorrhage [7-9], ischemic stroke [10,11], and trauma [12-15] and in patients with dural arteriovenous fistula (dAVF) $[16,17]$ and intracerebral $[18,19]$ and spinal $[20,21]$ neoplasms. However, within these studies, outcome measures are variable depending on the disease of interest, so a mix of elective and emergent patients are included and patients are derived from different institutions with variation in population demographics. A comparison of D-dimer biomarker prediction across different neurosurgical diseases, as one might come across in the average neurosurgical practice, has not been performed, limiting the ability to use this biomarker clinically. We aimed to explore the efficacy and accuracy with which Ddimers correlate with patient outcome. 


\section{Materials And Methods \\ Study sample}

After the Institutional Review Board approval, we undertook a retrospective chart review using bioinformatic search parameters to evaluate patients admitted by the neurosurgery service from March 2008 to August 2017 after the initiation of a D-dimer protocol for deep vein thrombosis (DVT) detection at our institution, which required admission D-dimer levels on all patients. A total of 1,918 discrete patient encounters involving 1,854 patients were observed, where encounters involved separate admissions and discharges as previously reported [22]. D-dimer levels were acquired from blood samples (test \#003057, reference range $0.0-0.4 \mu \mathrm{g} / \mathrm{ml}$; ARUP, Salt Lake City, UT) at the date of admission. Other laboratory markers on the date of admission included white blood count (WBC), prothrombin time (PT), partial thromboplastin time (PTT), erythrocyte sedimentation rate (ESR), C-reactive protein (CRP), and procalcitonin.

Patient variables were collected from the medical record by chart review. Measurements of clinical severity included admission Glasgow Coma Scale (GCS) score and American Society of Anesthesiologists (ASA) score when documented. A diagnosis of culture-positive infection or treatment with antibiotics was marked as a patient with infection. DVT evaluation by ultrasound or CT angiography was noted. Major surgical procedures, defined as the need for use of the operating room under general anesthesia, were noted. Patients were allocated into diagnostic categories including vascular, spine, trauma, tumor, multiple, and "other diagnoses" based on the primary reason for admission. Tumor and spine patients were primarily elective patients, trauma patients were emergent admissions, and vascular patients were a mix of elective and emergent patients. The primary outcome for this study was discharge disposition. Routine disposition was defined as home or home health, whereas nonroutine disposition was defined as a skilled nursing facility (SNF) or long-term acute care (LTAC), acute rehabilitation, other hospital, or death.

\section{Statistical analysis}

For continuous and discrete variables, means with standard deviation and percentages were calculated, respectively. Continuous and discrete variables were analysed by t-test and chi-squared test, respectively. The nonparametric Mann-Whitney U test was used to evaluate median value differences. For logistic regression models, the outcome of nonroutine disposition was analysed. Univariate logistic regression was performed to calculated relative risks (RRs) and $95 \%$ CIs. Variables with a $\mathrm{p}<0.1$ were entered into a multivariable model. A p-value $<0.05$ was considered significant. Statistics were analysed using SPSS Version 22.0 (IBM Corp., Armonk, NY).

\section{Results}

\section{Patient demographics}

A total of 1,918 patients (mean age $55.1 \pm 18.2$ years; $55.4 \%$ male) were identified (Table 1 ). The most common diagnostic subtype was vascular ( $n=519,27.1 \%)$, followed by trauma $(n=315,16.4 \%)$, multiple diagnoses $(n=301,15.7 \%)$, spine $(n=299,15.6 \%)$, tumor $(n=250,13.0 \%)$, and "other" $(n=234,12.2 \%)$. The mean length of stay (LOS) was $13.1 \pm 10.5$ days, and most patients had a nonroutine disposition $(\mathrm{n}=1323$, 69.0\%). The majority of nonroutine disposition was to rehabilitation ( $n=813,42.6 \%)$, followed by a SNF/LTAC $(\mathrm{n}=291,15.2 \%)$. The average admission D-dimer level was $4.83 \pm 7.78 \mu \mathrm{g} / \mathrm{ml}$. Mean D-dimer levels were significantly elevated and variable for trauma patients compared with the other diagnostic categories (oneway analysis of variance [ANOVA], Tukey post-hoc, $\mathrm{p}=0.0001$ ) (Figure $1 \mathrm{~A}$ ). 


\section{Cureus}

\begin{tabular}{|c|c|}
\hline Variable & Mean \pm SD or value ( $\%$ total) \\
\hline Age (years) & $55.1 \pm 18.2$ \\
\hline Sex (male) & $1062(55.4 \%)$ \\
\hline \multicolumn{2}{|l|}{ Race } \\
\hline Caucasian & $1555(81.1 \%)$ \\
\hline Other & $120(6.3 \%)$ \\
\hline Unknown & $98(5.1 \%)$ \\
\hline American Indian & 37 (1.9\%) \\
\hline Native Hawaiian/Pacific Islander & $36(1.9 \%)$ \\
\hline Black/African & $35(1.8 \%)$ \\
\hline Asian & $37(1.9 \%)$ \\
\hline \multicolumn{2}{|l|}{ Ethnicity } \\
\hline Non-Hispanic & $1624(84.7 \%)$ \\
\hline Hispanic & $136(7.1 \%)$ \\
\hline Unknown & $158(8.2 \%)$ \\
\hline Median ASA score & 3 \\
\hline Median GCS score & 8 \\
\hline \multicolumn{2}{|l|}{ Diagnosis subtype } \\
\hline Vascular & $519(27.1 \%)$ \\
\hline Trauma & $315(16.4 \%)$ \\
\hline Multiple & $301(15.7 \%)$ \\
\hline Spine & $299(15.6 \%)$ \\
\hline Tumor & $250(13.0 \%)$ \\
\hline Other & $234(12.2 \%)$ \\
\hline Length of stay (days) & $13.1 \pm 10.5$ \\
\hline \multicolumn{2}{|l|}{ Disposition } \\
\hline Routine & $415(21.6 \%)$ \\
\hline Nonroutine & $1503(78.4 \%)$ \\
\hline Infection/antibiotics & $704(36.7 \%)$ \\
\hline DVT treatment & $428(22.3 \%)$ \\
\hline Major surgical procedure in previous 365 days & $562(29.3 \%)$ \\
\hline Major surgical procedure within 120 days & $683(35.6 \%)$ \\
\hline Admission D-dimer level $(\mu \mathrm{g} / \mathrm{ml})$ & $4.83 \pm 7.78$ \\
\hline
\end{tabular}

\section{TABLE 1: Baseline demographics from 1,918 neurosurgical patient admissions}

ASA, American Society of Anesthesiologists; GCS, Glasgow Coma Scale; DVT, deep vein thrombosis 


\section{Cureus}
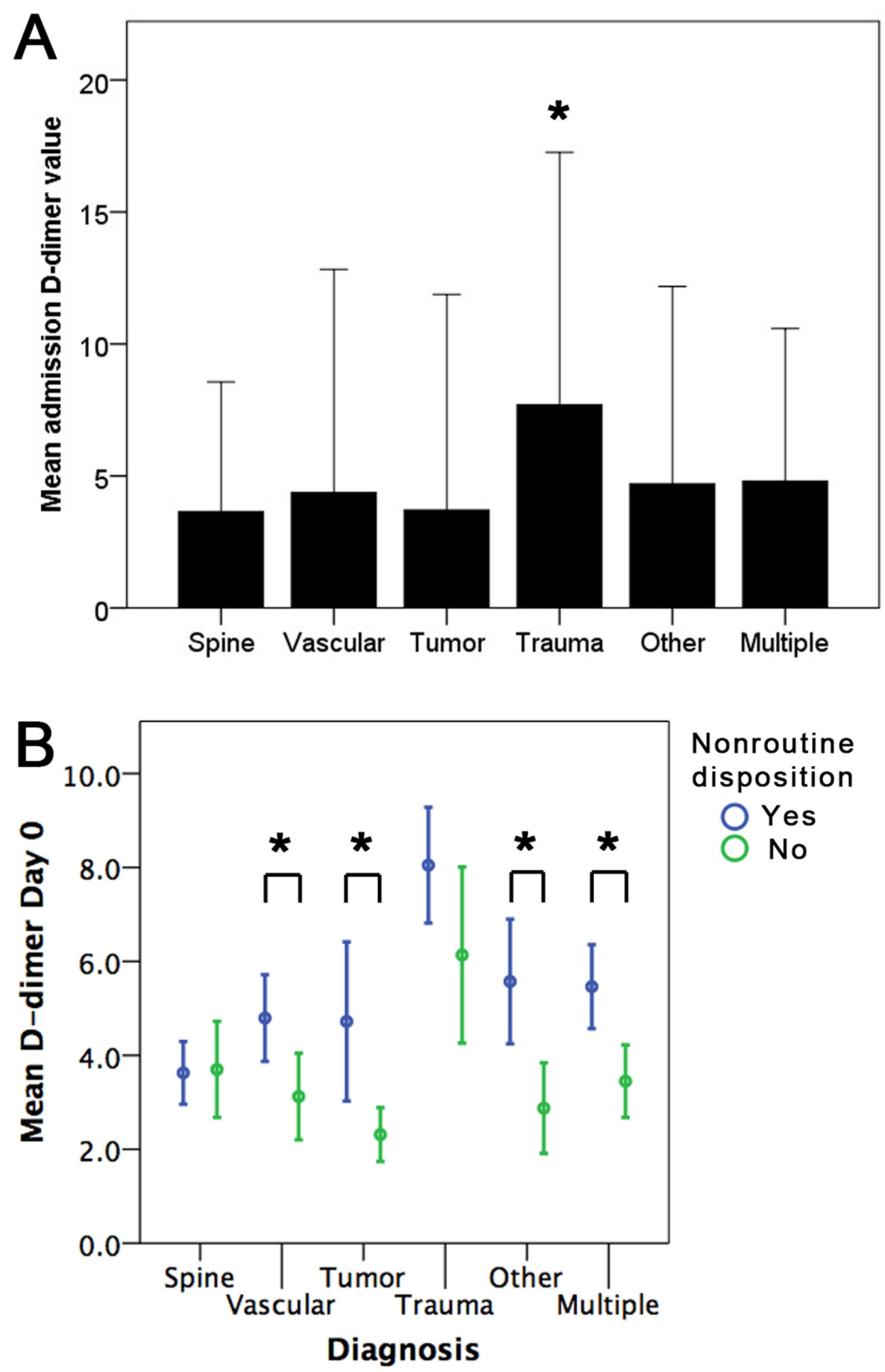

\section{FIGURE 1: D-dimer associations with patient outcomes}

(A) Mean admission D-dimer values differed between trauma patients and the other categories (one-way analysis of variance, Tukey post-hoc, $p=0.0001)$. (B) A significantly higher $D$-dimer level was seen for vascular $(p=0.05)$, tumor $(p=0.02)$, other $(p=0.01)$, and multiple diagnosis $(p=0.005)$ patients who had nonroutine discharge disposition. Spine and trauma patients did show differences in D-dimer levels for discharge dispositions. Error bars are $95 \% \mathrm{Cl}$.

${ }^{*} \mathrm{p}<0.05$.

\section{Patient outcomes}

Nonroutine discharge disposition showed an association with admission D-dimer levels across diagnosis types, except for spine or trauma patients (one-way ANOVA, Tukey post-hoc, $\mathrm{p}<0.05$ ) (Figure $1 B$ ). Univariate and multivariate logistic regression analysis compared clinical factors in patients with routine and nonroutine disposition (Table 2). Multivariate logistic regression demonstrated that age ( $\mathrm{RR}=1.026$, $\mathrm{p}=0.0001)$, trauma diagnosis $(R R=1.54, \mathrm{p}=0.04)$, greater $\operatorname{LOS}(\mathrm{RR}=1.09, \mathrm{p}=0.0001)$, infection $(\mathrm{R} R=1.5$, $p=0.001)$, $D$-dimer level $(R R=1.04, p=0.001)$, and $W B C(R R=1.02, p=0.05)$ were independently associated with a greater likelihood of nonroutine disposition. Non-Hispanic ethnicity ( $R R=0.64, p=0.04)$, higher GCS 
$(\mathrm{RR}=0.87, \mathrm{p}=0.0001)$, tumor diagnosis $(\mathrm{RR}=0.6, \mathrm{p}=0.004)$, and PTT $(\mathrm{RR}=0.984, \mathrm{p}=0.02)$ were associated with lower rates of nonroutine disposition.

\begin{tabular}{|c|c|c|c|c|}
\hline & \multicolumn{2}{|l|}{ Univariate } & \multicolumn{2}{|l|}{ Multivariate } \\
\hline & Unstandardized coeffıclents & p-value & Unstandardized coefficients & p-value \\
\hline Admission D-dimer & 0.06 & 0.006 & 0.2 & 0.05 \\
\hline Age & -0.2 & 0.0001 & -0.3 & 0.009 \\
\hline Sex & 0.04 & $0.07^{\mathrm{a}}$ & & \\
\hline Race & 0.08 & 0.001 & 0.1 & 0.2 \\
\hline Admission GCS & -0.4 & $0.0001^{b}$ & & \\
\hline Highest ASA score & 0.3 & $0.0001^{b}$ & & \\
\hline Treatment subtype & 0.032 & 0.2 & -0.08 & 0.8 \\
\hline Major surgical procedure within 120 days & 0.08 & 0.03 & -0.09 & 0.4 \\
\hline Disposition & 0.09 & 0.0001 & 0.5 & 0.0001 \\
\hline
\end{tabular}

\section{TABLE 2: Evaluation of association between clinical variables and discharge disposition}

ASA, American Society of Anesthesiologists; GCS, Glasgow Coma Scale

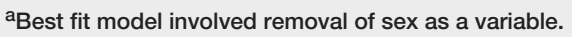

${ }^{\mathrm{b}}$ Colinear; GCS was removed from the multivariable model.

\section{Discussion}

\section{Study findings}

The results of this study suggest that higher admission D-dimer levels may be associated with worse patient disposition outcomes across various elective and emergent patients. This trend held for different categories of neurosurgical diagnoses, with the exception of trauma and spine, and after adjusting for various clinical risk factors such as DVT diagnosis, infection, and other elevated inflammatory markers. An approximately $3 \%$ greater likelihood of nonroutine disposition was seen for every unit increase in D-dimer levels on admission.

The implication for these findings is to stratify patient risk groups so that greater medical resources can be devoted earlier to high-risk patients. This may involve preemptive set-up for disposition, more aggressive physical therapy and rehabilitation, and discussion with family and staff regarding the expected longer treatment course for a high-risk patient. We did observe significant heterogeneity in D-dimer levels over this wide group of patients, likely as a reflection of the underlying physiology and disease differences. While it is premature to solely rely on D-dimers as a tool for prognostication, our data add to the existing body of literature for neurosurgical biomarkers and can be useful for the generation of DVT detection protocols at other institutions.

\section{D-dimer as a disease biomarker in neurosurgery}

D-dimers have previously been used to delineate outcome in a number of distinct diseases. In several small series, D-dimer levels in patients with subarachnoid hemorrhage or aneurysms have correlated with various outcome measures, including delayed cerebral ischemia, three-month Glasgow outcome scale, modified Rankin scale (mRS), and infections [3-6]. D-dimers have also been shown to correlate with poor outcomes in patients after ischemic stroke, including the Pediatric Stroke Outcome Measure and mRS [10,11], as well as thrombosis in dural arteriovenous fistulas [17]. Similarly, D-dimer levels correlate with worse outcomes in patients with traumatic brain injury (TBI), including posttraumatic cerebral infarction and hemorrhage [12,23-25], and with higher mortality in patients with gliomas or cranial or spinal metastatic disease [18$21,23,24,26]$.

Our study found a correlation of D-dimer levels and LOS with all diseases except for trauma. This was similar to only one other study, which found D-dimers within 24 hours were not correlated with the Glasgow 
Outcome Scale score after severe TBI [25]. However, in that study, the Glasgow Outcome Scale score did correlate with D-dimer after moderate TBI. Otherwise, we were able to show D-dimers could predict the increased risk of worsened disposition.

\section{Acute-phase proteins}

One reason D-dimers may be predictive of the outcome is because they serve as a biomarker of inflammation [27-29]. The APP response governs a cascade of pathological responses resulting in leukocytosis, elevation of acute reactive proteins (e.g., D-dimer, CRP, serum amyloid A, interleukins, tumor necrosis factor $\alpha$ ), as well as clinical responses (e.g., pyrexia, hormonal alterations, muscle protein depletion). A typical response after a stressful event is the elevation of reactive proteins within $24-48$ hours; however, the chronic inflammatory response may result from multiple events and may hinder physiologic recovery by potentially limiting tissue and wound healing, suppressing the immune system, and reducing physiologic reserve. This may likely increase susceptibility for patient complications and reduced mobility that can worsen disposition. Whereas several reactive proteins have well-defined specific clinical use (e.g., CRP and infection), the role of D-dimer remains to be better explored beyond a simple marker of thrombosis. All APPs show some non-specificity between infection and inflammation owing to the similar underlying molecular processes. Bridging the gap between serological laboratory changes and patient outcomes, which ultimately are complex and multifactorial, requires additional clinical stratification. D-dimer levels have not been predictive of prognosis for all neurosurgical diseases and it remains to be seen if this is simply the chosen study population or D-dimers serve as an epiphenomenon of inflammatory drivers that impact patient care $[25,30]$.

\section{Study limitations}

One limitation involves the use of disposition as a common outcome. Although disposition is a complex end-point, impacted by disease severity, clinical treatment course, and socioeconomic factors, it nonetheless remains an important outcome related to treatment and cost. Disposition was comparable across different diseases and can be objectively verified by other researchers. Another limitation of this study involved the difficulty in accounting for disease severity across different disease groups. Adjusting patients for different disease severity was attempted using GCS, ASA score, disease subgroups, major surgical procedure, and presence of an infection or DVT. However, application of these variables differs among patients due to different standards in documentation for patient diseases. For example, GCS is not commonly acquired on our vascular patients as compared with a trauma patient. Further study of D-dimer biomarkers may benefit from replication of these findings and a prospective follow-up with additional clinical variables and outcome measurements. Although we did not specifically look at a D-dimer cutoff predicting a higher likelihood of nonroutine disposition, our prior studies did demonstrate that D-dimer levels of $\geqslant 2.5 \mu \mathrm{g} / \mathrm{ml}$ predicted a $30 \%$ higher likelihood of venous thromboembolism [18]. Future studies can potentially be helpful with generating cutoff values and clinical scores to predict outcome while using Ddimers.

\section{Conclusions}

Higher admission D-dimer levels were independently associated with poorer discharge prognosis in a sample of neurosurgical patients, even after adjusting for disease severity and other clinical factors. A 3\% greater relative risk of nonroutine disposition was seen for every one-unit increase in D-dimer levels. However, additional follow-up studies will be needed to objectively evaluate the ability of D-dimers to correlate with patient outcomes and improve predictive models. A better pathophysiological understanding of the inflammatory response in neurosurgical patients will also be necessary to progress from simply predicting outcome to intervening and improving treatments.

\section{Additional Information}

\section{Disclosures}

Human subjects: Consent was obtained by all participants in this study. University of Utah issued approval IRB_00074046. This study has been approved by the University of Utah Institutional Review Board. Animal subjects: All authors have confirmed that this study did not involve animal subjects or tissue. Conflicts of interest: In compliance with the ICMJE uniform disclosure form, all authors declare the following: Payment/services info: All authors have declared that no financial support was received from any organization for the submitted work. Financial relationships: All authors have declared that they have no financial relationships at present or within the previous three years with any organizations that might have an interest in the submitted work. Other relationships: All authors have declared that there are no other relationships or activities that could appear to have influenced the submitted work.

\section{Acknowledgements}

We thank Kristin Kraus, MSc, for editorial support, D. Ryan Ormond, MD, and Patrick W. Hosokawa, MS, for discussion of the paper, and Ramkiran Gouripeddi, MS, MBBS, and Ryan Butcher, BS, for support with data acquisition. 


\section{References}

1. Pulivarthi S, Gurram MK: Effectiveness of D-dimer as a screening test for venous thromboembolism: an update. N Am J Med Sci. 2014, 6:491-499. 10.4103/1947-2714.143278

2. Wells PS, Anderson DR, Rodger M, et al.: Evaluation of D-dimer in the diagnosis of suspected deep-vein thrombosis. N Engl J Med. 2003, 349:1227-1235. 10.1056/NEJMoa023153

3. Boluijt J, Meijers JC, Rinkel GJ, Vergouwen MD: Hemostasis and fibrinolysis in delayed cerebral ischemia after aneurysmal subarachnoid hemorrhage: a systematic review. J Cereb Blood Flow Metab. 2015, 35:724733. $10.1038 / \mathrm{jcbfm} .2015 .13$

4. Ilveskero S, Juvela S, Siironen J, Lassila R: D-dimer predicts outcome after aneurysmal subarachnoid hemorrhage: no effect of thromboprophylaxis on coagulation activity. Neurosurgery. 2005, 57:16-24. 10.1227/01.NEU.0000163085.48999.D6

5. Liu JH, Li XK, Chen ZB, Cai Q, Wang L, Ye YH, Chen QX: D-dimer may predict poor outcomes in patients with aneurysmal subarachnoid hemorrhage: a retrospective study. Neural Regen Res. 2017, 12:2014-2020. 10.4103/1673-5374.221158

6. Fukuda H, Lo B, Yamamoto Y, Handa A, Yamamoto Y, Kurosaki Y, Yamagata S: Plasma D-dimer may predict poor functional outcomes through systemic complications after aneurysmal subarachnoid hemorrhage. J Neurosurg. 2017, 127:284-290. 10.3171/2016.5.JNS16767

7. Chen CW, Wu EH, Huang J, Chang WT, Ao KH, Cheng TJ, Yang W: Dynamic evolution of D-dimer level in cerebrospinal fluid predicts poor outcome in patients with spontaneous intracerebral hemorrhage combined with intraventricular hemorrhage. J Clin Neurosci. 2016, 29:149-154. 10.1016/i.jocn.2015.10.036

8. Cho TG, Lee JC, Park SW, Chung C, Nam TK, Hwang SN: Relationship between systemic thrombogenic or thrombolytic indices and acute increase of spontaneous intracerebral hemorrhage. J Cerebrovasc Endovasc Neurosurg. 2014, 16:159-165. 10.7461/jcen.2014.16.3.159

9. Hu X, Fang Y, Ye F, Lin S, Li H, You C, Liu M: Effects of plasma D-dimer levels on early mortality and longterm functional outcome after spontaneous intracerebral hemorrhage. J Clin Neurosci. 2014, 21:1364-1367. 10.1016/j.jocn.2013.11.030

10. Zhang J, Liu L, Tao J, Song Y, Fan Y, Gou M, Xu J: Prognostic role of early D-dimer level in patients with acute ischemic stroke. PLoS One. 2019, 14:e0211458. 10.1371/journal.pone.0211458

11. Goldenberg NA, Jenkins S, Jack J, et al.: Arteriopathy, D-dimer, and risk of poor neurologic outcome in childhood-onset arterial ischemic stroke. J Pediatr. 2013, 162:1041-1046.E1. 10.1016/j.jpeds.2012.11.035

12. Chen H, Xue LX, Guo Y, et al.: The influence of hemocoagulation disorders on the development of posttraumatic cerebral infarction and outcome in patients with moderate or severe head trauma. Biomed Res Int. 2013, 2013:685174. 10.1155/2013/685174

13. Juratli TA, Zang B, Litz RJ, et al.: Early hemorrhagic progression of traumatic brain contusions: frequency, correlation with coagulation disorders, and patient outcome: a prospective study. J Neurotrauma. 2014, 31:1521-1527. 10.1089/neu.2013.3241

14. Sugimoto K, Suehiro E, Shinoyama M, et al.: D-dimer elevation as a blood biomarker for detection of structural disorder in mild traumatic brain injury. J Neurotrauma. 2017, 34:3245-3248. 10.1089/neu.2017.5240

15. Yuan F, Ding J, Chen H, et al.: Predicting outcomes after traumatic brain injury: the development and validation of prognostic models based on admission characteristics. J Trauma Acute Care Surg. 2012, 73:137-145. 10.1097/TA.0b013e31824b00ac

16. Gerlach R, Boehm-Weigert M, Berkefeld J, Duis J, Raabe A, Seifert V, Marquardt G: Thrombophilic risk factors in patients with cranial and spinal dural arteriovenous fistulae. Neurosurgery. 2008, 63:693-699. 10.1227/01.NEU.0000325730.77263.7E

17. Izumi T, Miyachi S, Hattori K, Iizuka H, Nakane Y, Yoshida J: Thrombophilic abnormalities among patients with cranial dural arteriovenous fistulas. Neurosurgery. 2007, 61:262-269. 10.1227/01.NEU.0000255529.46092.7C

18. Navone SE, Guarnaccia L, Locatelli M, et al.: Significance and prognostic value of the coagulation profile in patients with glioblastoma: implications for personalized therapy. World Neurosurg. 2019, 121:e621-e629. 10.1016/j.wneu.2018.09.177

19. Hoke M, Dieckmann K, Koppensteiner R, Schillinger M, Marosi C, Mlekusch W: Prognostic value of plasma d-dimer levels in patients with glioblastoma multiforme - results from a pilot study. Wien Klin Wochenschr. 2011, 123:199-203. 10.1007/s00508-011-1556-9

20. Li B, Zhang H, Zhou P, et al.: Prognostic significance of pretreatment plasma D-dimer levels in patients with spinal chordoma: a retrospective cohort study. Eur Spine J. 2019, 28:1480-1490. 10.1007/s00586-01805872-4

21. Yang M, Xu W, Liu T, et al.: Development and validation of a novel survival prediction model in patients with spinal metastasis from non-small cell lung cancer. Spine (Phila Pa 1976). 2019, 44:246-257. 10.1097/BRS.0000000000002816

22. Karsy M, Azab MA, Harper J, et al.: Evaluation of a D-dimer protocol for detection of venous thromboembolism. World Neurosurg. 2020, 133:e774-e783. 10.1016/j.wneu.2019.09.160

23. Tian HL, Chen H, Wu BS, et al.: D-dimer as a predictor of progressive hemorrhagic injury in patients with traumatic brain injury: analysis of 194 cases. Neurosurg Rev. 2010, 33:359-366. 10.1007/s10143-010-0251-z

24. Berger RP, Fromkin J, Rubin P, Snyder J, Richichi R, Kochanek P: Serum D-dimer concentrations are increased after pediatric traumatic brain injury. J Pediatr. 2015, 166:383-388. 10.1016/j.jpeds.2014.10.036

25. Gupta G, Wadhwa C, Garg R, Dhaiya RS, Kaushal RK: Impact of coagulation profile on outcome of head injury. J Clin Diagn Res. 2016, 10:PC04-PC06. 10.7860/JCDR/2016/14638.7029

26. Yuan F, Ding J, Chen H, et al.: Predicting progressive hemorrhagic injury after traumatic brain injury: derivation and validation of a risk score based on admission characteristics. J Neurotrauma. 2012, 29:21372142. 10.1089/neu.2011.2233

27. Gruys E, Toussaint MJ, Niewold TA, Koopmans SJ: Acute phase reaction and acute phase proteins . J Zhejiang Univ Sci B. 2005, 6:1045-1056. 10.1631/jzus.2005.B1045 


\section{Cureus}

28. Jain S, Gautam V, Naseem S: Acute-phase proteins: as diagnostic tool . J Pharm Bioallied Sci. 2011, 3:118127. 10.4103/0975-7406.76489

29. Watterson C, Lanevschi A, Horner J, Louden C: A comparative analysis of acute-phase proteins as inflammatory biomarkers in preclinical toxicology studies: implications for preclinical to clinical translation. Toxicol Pathol. 2009, 37:28-33. 10.1177/0192623308329286

30. Squizzato A, Ageno W, Finazzi S, Mera V, Romualdi E, Bossi A, Venco A: D-dimer is not a long-term prognostic marker following acute cerebral ischemia. Blood Coagul Fibrinolysis. 2006, 17:303-306. 10.1097/01.mbc.0000224850.57872.d0 